\title{
ZOIL: A Design Paradigm and Software Framework for Post-WIMP Distributed User Interfaces
}

\author{
Michael Zöllner, Hans-Christian Jetter, and Harald Reiterer
}

\begin{abstract}
We introduceZOIL (Zoomable Object-Oriented Information Landscape), a design paradigm and software framework for post-WIMP distributed, zoomable and object-oriented user interfaces. This paper presents ZOIL's design principles, functionality and software patterns to share them with other researchers. Additionally, ZOIL's implementation as a software framework for C\# and WPF (Windows Presentation Foundation) is available as open source under the BSD License for scientific and commercial projects (see http://zoil.codeplex.com).
\end{abstract}

Keywords Zoomable user interfaces $\bullet$ Post-WIMP user interfaces $\bullet$ Object-oriented user interfaces $\bullet$ Software framework

\subsection{Introduction}

To this day, most DUI research has focused on the distribution of standard Graphical User Interfaces (GUIs) and their widgets (e.g. [2, 14]). In this paper, we introduce ZOIL, a design paradigm and software framework targeted at post-WIMP (post"Windows Icons Menus Pointer") multi-touch or tangible UIs that are distributed over multiple devices and displays. Designing and implementing such DUIs is particularly challenging: While the essence and the building blocks of a standard desktop "WIMP" GUI are well established, there is no such concise pattern or blueprint for the post-WIMP world. Thus, there are no established design principles, UI toolkits and programming models available. As a consequence, post-WIMP DUIs

\footnotetext{
M. Zöllner $(\bowtie) \bullet$ H.-C. Jetter $\bullet$ H. Reiterer

Human-Computer Interaction Group, University of Konstanz, Konstanz, Germany e-mail: michael.zoellner@uni-konstanz.de; hans-christian.jetter@uni-konstanz.de; harald.reiterer@uni-konstanz.de
} 
require great expertise in designing and programming many heterogeneous interaction modalities and soft- and hardware technologies. We created ZOIL to better support designers and developers in this task. The ZOIL design paradigm suggests patterns of solution as heuristics for choosing suitable conceptual models, visualizations and interaction techniques. The ZOIL software framework then facilitates their efficient implementation $[8,18]$.

\subsection{The ZOIL Design Paradigm}

Applications following the ZOIL design paradigm integrate all information items from the application domain with all their connected functionality and with their mutual relations into a single visual workspace under a consistent interaction model [10]. This visual workspace is called the "information landscape" and its interaction model follows the principles of Zoomable and Object-Oriented User Interfaces (ZUIs [16, 20], OOUIs [3, 13]): All information items are integrated into a single object-oriented domain model and appear as visual objects at one or more places in the information landscape. This way, the domain model becomes directly user-accessible similar to the "naked objects" pattern [15]. Unlike the desktop metaphor, the information landscape is not limited to the visible screen size but resembles a canvas of infinite size and resolution for persistent visualspatial information management. In the information landscape all items and their functionality can be accessed directly in an object-oriented manner [3] without the use of application windows simply by panning to the right spot in the information landscape and zooming in [20]. ZOIL thereby uses "semantic zooming" [16] which means that the geometric growth in display space is not only used to render more details, but to reveal more and semantically different content and all available functionality "on-the-spot". This smooth transition between iconic representation, meta-data, and full-text/full-functionality prevents the problems of information overload and disorientation which are caused by traditional WIMP approaches with multiple overlaying windows or occluding renderings of detailson-demand.

Although the previous description focuses mainly on a single user's view, ZOIL's key strength lies in its great flexibility in distributed and collaborative scenarios: Using ZOIL, DUIs can be designed either as homogeneous or heterogeneous [2], i.e. the UIs can either offer multiple instances of the same user interface at different sites or the functionality provided at each site may be different. This flexibility is achieved by the real-time distribution of the object-oriented data model of the information landscape to all devices in an interactive space (Fig. 10.1). Each connected client can render an arbitrary part of the shared landscape, so that users can navigate and manipulate it with their client independently. Thereby users can also use the full breadth of the available device-specific input and output modalities. A connected client is also free to use other visualization styles, e.g. for a device-, user-, or taskspecific representation of the landscape's content. 


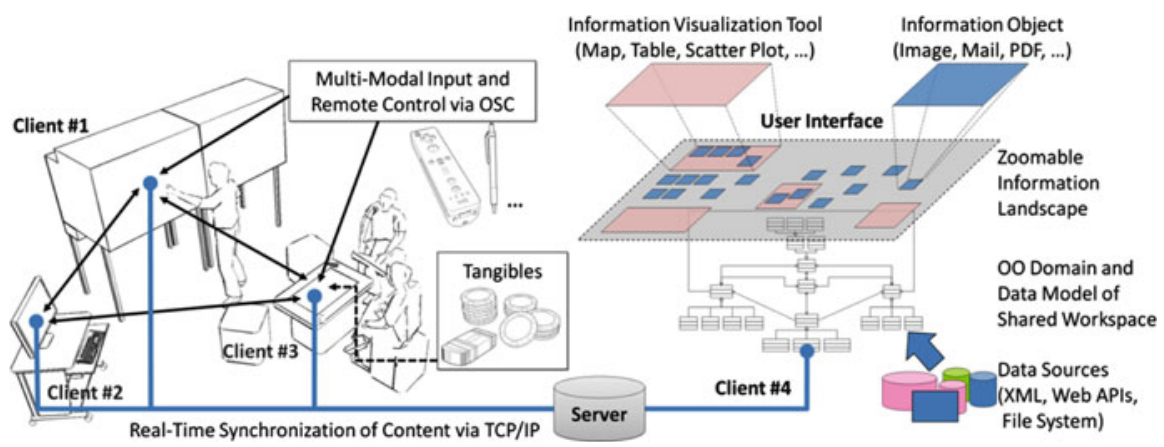

Fig. 10.1 A ZOIL-based multi-user, multi-surface, and multi-device interactive space (left). The right section shows the architecture and user interface of a ZOIL client application in detail

\subsection{The ZOIL Software Framework}

Implementing a post-WIMP distributed user interface following the ZOIL design paradigm without tool support is a difficult task that demands a wide range of expertise ranging from distributed databases to vector-based rendering of UI controls. To facilitate ZOIL's application in practice, we have developed the ZOIL software framework [19] that provides the necessary tool support as an extensible collection of software components and classes for C\#/WPF. Although individual aspects of ZOIL's implementation are already covered by existing frameworks such as Piccolo [1] or DiamondSpin [21], incompatibilities between languages, UI frameworks, and device SDKs have led us to build a new custom framework for our purposes. The framework has already been used for the implementation of various user interfaces (e.g. [8, 9, 17]) and its API usability was evaluated in a longitudinal study [5]. In the light of the framework's volume and broad scope, we focus our description here on its defining features to share our most important experiences. We describe those essential software patterns and architectures from the framework that can also be employed by other researchers in their own projects to implement advanced designs of post-WIMP distributed user interfaces.

\subsubsection{Client-Server Architecture with Transparent Persistence}

For realizing the distribution from Fig. 10.1, ZOIL employs a central database server for real-time synchronization and persistence. Following the nature of the real world, ZOIL's objects and information landscape do not lose their individual state, properties, or location when disappearing from the screen or after closing the application. Furthermore they are synchronized with all other connected clients in real-time to allow for multi-device and multi-user interaction. We have decided to distribute the UI by synchronizing the data model of the shared visual workspace instead of the UI's visual appearance using protocols on the "pixel level" such as VNC or RDP. Thus, the 


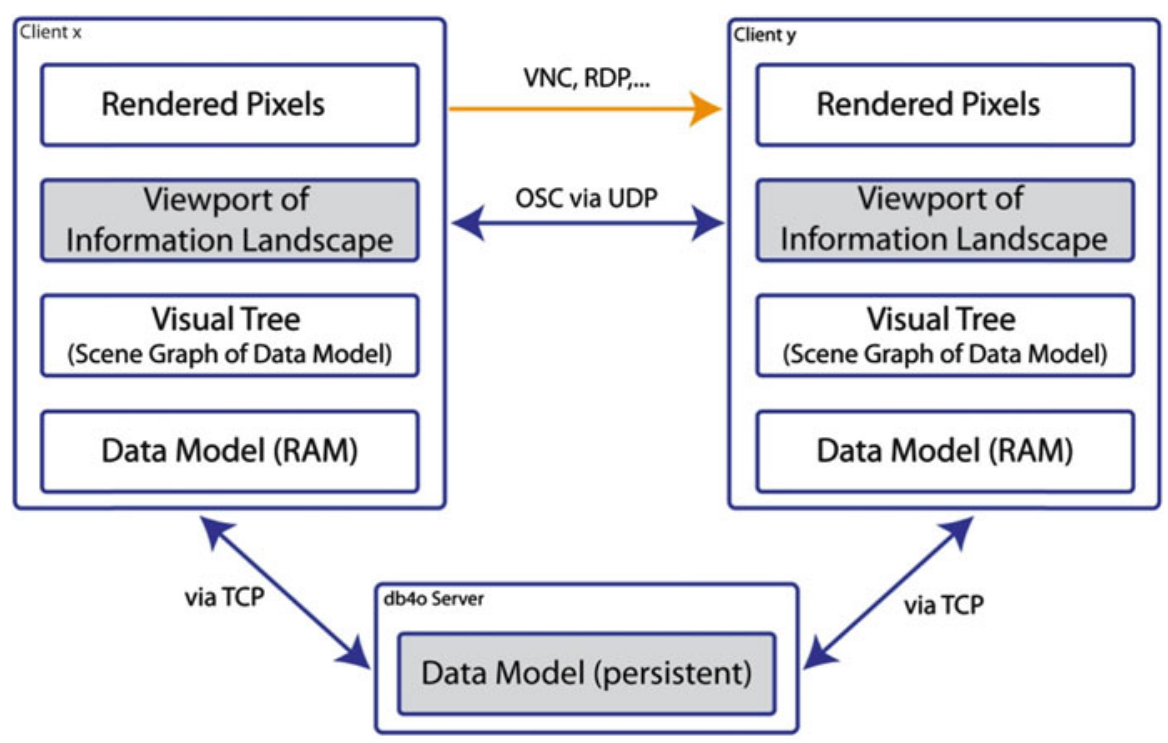

Fig. 10.2 Overview of ZOIL's distribution capabilities

overall network load of a ZOIL setup is relatively small, since only small changes of the data model are transmitted (Fig. 10.2). To achieve this, the ZOIL software framework uses the object database db4o [4] with its Transparent Persistence (TP) mechanism. The aim of TP is to enable programmers to access or change persistent data in their application with the same ease as non-persistent data models in main memory. This means that with TP and db4o, ZOIL developers are not concerned with persistence at all since persistence simply becomes a natural property of all ZOIL objects. Using TP, programmers can simply change the states and locations of objects on the client-side using standard C\# code. Invisible to the programmer, all changes are then observed by db4o's TP implementation. It collects all changes and transmits them via TCP/IP to the database server in regular intervals (typically $100 \mathrm{~ms}$ ). The server persists the new state of the landscape and informs all other clients about the changes which are then automatically retrieved and executed on the remote clients within 100 $300 \mathrm{~ms}$ (in typical setups). Thereby the use of the object database db4o instead of traditional Object-Relational Mapping libraries (e.g. Hibernate) also facilitates iterative design, since changes in the objects' class definitions in C\# are directly recognized by $\mathrm{db} 4 \mathrm{o}$ without the need to update external XML mappings.

\subsubsection{Model-View-ViewModel Pattern (MVVM)}

TP offers low-threshold persistency for data objects, but it cannot be applied directly on WPF's visual hierarchies or user controls. Moreover such exact replications of widgets or controls would not be desirable in ZOIL, since each client should provide the user 
with an individual view on the landscape's shared data model, so that users can independently navigate and manipulate content. For this reason, the ZOIL framework uses the MVVM Pattern [7] to provide a novel way of separating the persistent data model of an object from the non-persistent view of the object in the landscape. Using TP, each object's model is shared via the server with all others clients, but the corresponding view is not shared and only resides on the client-side. As soon as a model is created (or destroyed) in a local or remote client, the corresponding views on all other clients are created (or destroyed) accordingly. The key advantage of integrating MVVM in ZOIL over using a traditional MVC approach lies in the ViewModel, which plays the role of an adapter between the XAML (Extensible Application Markup Language) markup of the view and the C\# model by tailoring the data of the model to a view-friendly format. Properties and commands of the ViewModel are bound via two-way data binding to the view. Model and ViewModel always stay synchronized, so changes in the model get routed to the view and vice versa. Using MVVM, views can also be defined more conveniently in declarative XAML, without the need for C\# code.

\subsubsection{Declarative Definition of Zoomable UI Components}

One key feature of our framework is the comprehensive support for authoring and implementing rich ZUIs with semantic zooming using WPF and its declarative XAML language. Unlike Java's SWT, Swing, or WinForms, WPF natively supports vectorbased and hardware-accelerated rendering of controls. This enables ZOIL designers to use the full range of WPF controls (e.g. sliders, player controls) in ZUIs without pixelation or the need for wrapper classes as in [1]. This also allows programmers to easily integrate media content such as video streams or $3 \mathrm{~d}$ models and increases the prototyping efficiency in comparison to other hardware-accelerated graphics APIs without any or only very basic sets of user controls (e.g. OpenGL, DirectX).

A further advantage of WPF is the possibility to separate the object's implementation logic in C\# from its visual design in XAML. This allows designers to use a low-threshold HTML-like language and visual editors for defining an object's appearance. As a consequence, ZOIL supports typical designer-developer workflows and lowers the threshold for non-programmers to use the framework. Following this declarative paradigm, we have extended the available set of controls and behaviors in XAML with a collection of ZOIL-specific components that enables designers to define semantic zooming and other behaviors without the need for procedural C\# code (Code Sample 10.1). The different appearances are selected by ZOIL's ZComponentFrames container depending on the current display size and are exchanged using a smooth zooming and opacity animation.

\subsubsection{Attached Behavior Pattern (ABP)}

For designing object-oriented interaction it is not sufficient to define an object's visual appearance alone. Objects also have to be assigned the desired behaviors: 


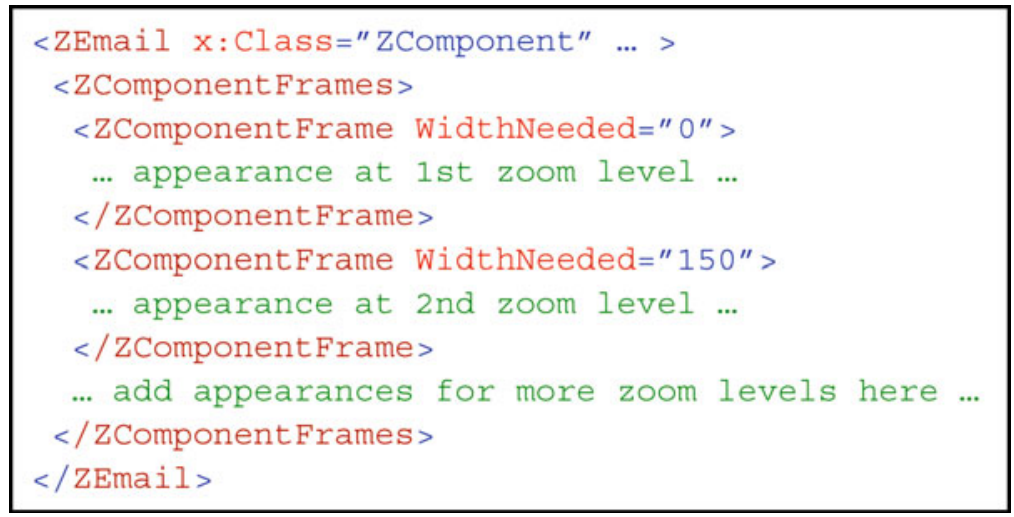

Code Sample 10.1 XAML code for defining the semantic zoom of an email object

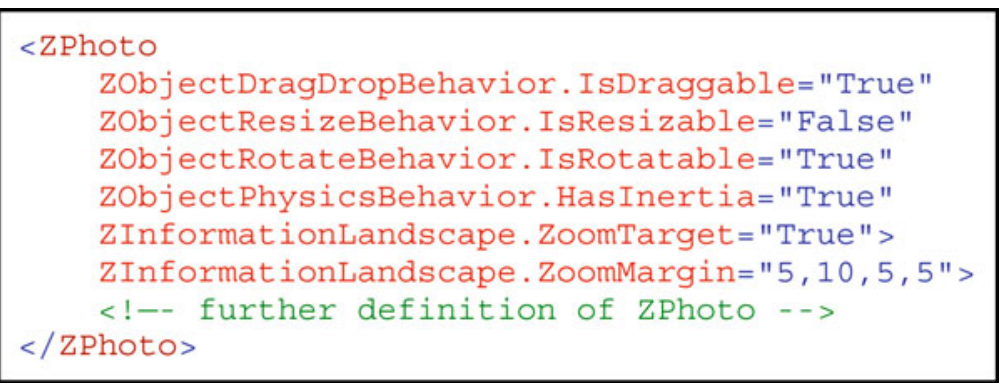

Code Sample 10.2 XAML for making ZPhoto a draggable, resizable, and rotatable zoom target

Can an object be dragged, resized, or rotated using multi-touch manipulations? Does an object simulate physical behavior such as inertia or friction during these manipulations? Is the object a zoom target, so that a click or tap on it starts a zooming animation into the object until it covers the screen? Or should there be a small margin remaining around the object? To help designers to easily assign such behaviors to an object without reinventing the wheel for every type, our framework makes extensive use of the Attached Behavior Pattern (ABP) [6]. ABP encapsulates behaviors into a class outside the object's class hierarchy and allows it to be applied on classes or individual instances of objects. When using WPF this threshold can be further lowered using XAML instead of C\# (Code Sample 10.2). We believe that the Attached Behavior Pattern introduces a very natural view of interactive behavior into post-WIMP programming that facilitates iterative design without the need for changes in procedural C\# code or class hierarchies. Furthermore by using ABP, a framework can provide designers with an extensible library of potentially useful behaviors that can be easily assigned in visual editors. 


\subsubsection{Input Device and Client-Client Communication with OSC}

The ZOIL framework provides simple ways to connect to multi-modal input libraries or other kinds of input device middleware $[11,12]$. Applications can use the stateless UDP-based OSC protocol to connect to open source tools like Squidy [11] that facilitate the integration of external post-WIMP devices such as Anoto pens or the Nintendo Wiimote. OSC has several advantages for prototyping: First, clients stay tolerant to unavailable devices or changing infrastructures during their lifetime, since no permanent connections have to be established. Second, UDP packets can be broadcasted to all clients in the subnet and therefore sending to specific IP addresses is unnecessary. Instead, packet destinations can be specified above the network layer using OSC addresses. This is desirable in early phases of prototyping and experimentation where devices are often added or removed. In some scenarios (e.g. in an augmented meeting room) it might be important to tightly couple two or more clients' views. By sending OSC commands between clients it is possible to control the current camera or viewport of a remote client from a local client. This allows scenarios of collocated collaboration between multiple users and devices. For example, a view on a region of the landscape can be transferred from a personal device to a public large display or vice versa. OSC can also be used to implement an overview and detail coupling between two clients.

\subsection{Conclusion}

We have introduced the ZOIL design paradigm and software framework. ZOIL is unique in its focus on distributed post-WIMP user interfaces that are based on a real-time synchronized multi-user/multi-display/multi-device visual workspace. A ZOIL setup therefore follows Melchior et al.'s notion of a Distributed User Interface: "A DUI consists of a UI having the ability to distribute parts or whole of its components across multiple monitors, devices, platforms, displays, and/or users" [14]. Given the broad design space of post-WIMP user interfaces, a generic design or implementation of "optimal" distribution is very difficult, if not impossible. In a ZOIL setup, it is possible to leave such decisions to each connected client, thereby avoiding the risk of losing usability or functionality and getting the most out of every client's input and output modalities. While ZOIL is inherently multi-monitor, multi-display, multi-device and multi-user, it is not truly multi-platform yet, since it only supports the MS Windows platform. We will evaluate if future versions of ZOIL can leverage Microsoft's Silverlight platform to come closer to a multiplatform solution. 


\section{References}

1. Bederson, B., Grosjean, J., Meyer, J.: Toolkit design for interactive structured graphics. IEEE Trans. Softw. Eng. 30, 535-546 (2004)

2. Bharat, K., Brown, M.H.: Building distributed, multi-user applications by direct manipulation. In: Proceedings of UIST 1994, pp. 71-80. Marina del Rey, New York (1994)

3. Collins, D.: Designing Object-Oriented User Interfaces, 1st edn. Benjamin-Cummings Publishing, Redwood City (1994)

4. db4o. Native java \& . net open source object database. http://www.db4o.com. Accessed 14 June 2011

5. Gerken, J., Jetter, H.-C., Zoellner, M., Mader, M., Reiterer, H.: The concept maps method as a tool to evaluate the usability of apis. In: Proceedings of CHI 2011, pp. 3373-3382. Vancouver, New York (2011)

6. Gossman, J.: The attached behavior pattern. http://blogs.msdn.com/b/johngossman/ archive/2008/05/07/the-attached-behavior-pattern.aspx. Accessed 14 June 2011

7. Gossman, J.: Introduction to the model view viewmodel pattern. http://blogs.msdn.com/b/ johngossman/archive/2005/10/08/478683.aspx. Accessed 14 June 2011

8. Jetter, H.-C., Gerken, J., Zöllner, M., Reiterer, H.: Model-based design and prototyping of interactive spaces for information interaction. In: Proceedings of HCSE 2010, pp. 22-37. Reykjavik, Berlin (2010)

9. Jetter, H.-C., Gerken, J., Zoellner, M., Reiterer, H., Milic-Frayling, N.: Materializing the query with facet-streams - a hybrid surface for collaborative search on tabletops. In: Proceedings of CHI 2011, pp. 3013-3022. Vancouver, New York (2011)

10. Jetter, H.-C., König, W.A., Gerken, J., Reiterer, H.: Zoil - a cross-platform user interface paradigm for personal information management. Personal Information Management: The disappearing desktop (a CHI 2008 Workshop)

11. König, W., Rädle, R., Reiterer, H.: Interactive design of multimodal user interfaces - reducing technical and visual complexity. J. Multimod. User Interfaces 3(3), 197-213 (2010)

12. Lawson, J., Al-Akkad, A., Vanderdonckt, J., Macq, B.: An open source workbench for prototyping multimodal interactions based on off-the-shelf heterogeneous components. In: Proceedings of EICS 09, Pittsburgh, pp. 245-254. Pittsburgh, New York (2009)

13. Mandel, T.: The GUI-OOUI War, Windows vs. OS/2: The Designer's Guide to HumanComputer Interfaces. Van Nostrand Reinhold, New York (1994)

14. Melchior, J., Grolaux, D., Vanderdonckt, J., Van Roy, P.: A toolkit for peer-to-peer distributed user interfaces: Concepts, implementation, and applications. In: Proceedings of EICS 2009, pp. 69-78. Pittsburgh, New York (2009)

15. Pawson, R.: Naked objects. PhD thesis, Trinity College, Dublin (2004)

16. Perlin, K., Fox, D.: Pad: An alternative approach to the computer interface. In: Proceedings of SIGGRAPH 93, pp. 57-64. Anaheim, New York

17. Project Deskpiles. http://research.microsoft.com/en-us/projects/deskpiles. Accessed 14 June 2011

18. Project Permaedia/ZOIL. http://hci.uni-konstanz.de/permaedia. Accessed 14 June 2011

19. Project ZOIL on Codeplex. http://zoil.codeplex.com. Accessed June 14, 2011

20. Raskin, J.: The Humane Interface: New Directions for Designing Interactive Systems. ACM Press/Addison-Wesley Publishing, Reading/Boston, New York (2000)

21. Shen, C., Vernier, F., Forlines, C., Ringel, M.: Diamondspin: an extensible toolkit for aroundthe-table interaction. In: Proceedings of CHI 2004, pp. 167-174. Vienna, New York (2004) 